\title{
ARTIGO REVISÃ0: Colagem de Folhas Cerâmicas
}

\section{(REVIEW ARTICLE: Tape Casting)}

\author{
Dachamir Hotza* \\ Technische Universität Hamburg-Harburg \\ Arbeitsbereich Technische Keramik \\ Postfach 901052, D-21071 Hamburg, Alemanha
}

\begin{abstract}
Resumo
A colagem de folhas cerâmicas (tape casting) é uma técnica de fabricação de peças cerâmicas de espessura fina e grande área superficial, usadas principalmente na indústria eletrônica como substratos de circuitos e capacitores de multicamadas. $\mathrm{O}$ processo consiste essencialmente na formação de uma barbotina e na colagem desta por meio de uma lâmina niveladora sobre uma superfície, geralmente móvel. Depois da secagem, é produzida uma folha flexível e resistente que pode então ser sinterizada. Neste artigo de revisão, são discutidos alguns conceitos teóricos envolvidos na formulação da barbotina, bem como aspectos tecnológicos do processo de colagem de folhas cerâmicas.
\end{abstract}

\section{INTRODUÇÃO}

A colagem de folhas (tape casting) é uma técnica utilizada para a fabricação de peças cerâmicas planas, finas (de 0,025 a $1 \mathrm{~mm}$ ), com áreas superficiais grandes [1,2]. Essa técnica tem despertado crescente interesse, especialmente em aplicações relacionadas à indústria eletrônica. As principais aplicações atuais incluem capacitores de multicamadas (MLC: multilayered capacitors) de titanato de bário, [3-5] substratos para circuitos eletrônicos e pacotes cerâmicos de multicamadas (MLCP: multilayered ceramic packages) de alumina, [6-11] de nitreto de alumínio [10-13] e de vitrocerâmica [10, 11].

A colagem de folhas foi originalmente desenvolvida nos anos 40 como um método de formar placas finas de materiais piezoelétricos e capacitores [14]. A primeira patente do processo, publicada em 1952, descreve o uso de barbotinas aquosas e nãoaquosas utilizando-se um dispositivo móvel provido de uma lâmina (processamento descontínuo) [15]. Esta tecnologia foi melhorada em uma patente publicada em 1961, na qual o processamento contínuo é introduzido [16]. Avanços no processo de colagem de folhas cerâmicas, tanto em formulações quanto em equipamentos têm continuado a partir da década de 70 , quando as primeiras revisões sobre o processo foram publicadas $[7,8]$.

O processo consiste basicamente na preparação de uma barbotina de um pó cerâmico, e na colagem dessa barbotina sobre

\footnotetext{
*Endereço atual: LabMat - Centro Tecnológico - Universidade Federal de Santa Catarina - Caixa Postal 476, 88040-900 Florianópolis - SC e-mail: hotza@labmat.ufsc.br
}

\section{Abstract}

Tape casting is a fabrication technique of thin ceramic sheets with large surface areas which are used mainly in the electronic industry as substrates and multilayered capacitors. The process consists essentially in forming a slurry and in casting it through a doctor blade on a generally moving surface. After drying, a flexible, resistant tape is produced which can be then sintered. In this review some theoretical concepts involved in the slurry formulation, as well as technical points of the tape casting process are discussed.

uma superfície. A barbotina é formada por solventes (água ou líquidos orgânicos), dispersantes, ligantes e plastificantes. Depois da evaporação do solvente, resta um filme flexível, que é separado da superfície e pode ser enrolado, cortado, perfurado, estampado ou laminado. Posteriormente, o material é tratado termicamente para a eliminação das substâncias orgânicas e sinterizado. A Fig. 1 apresenta um fluxograma das etapas fundamentais do processo.

A colagem de folhas cerâmicas é, em certos aspectos, um processo assemelhado à colagem de barbotina (slip casting). Muitos dos problemas relativos ao processamento de suspensões cerâmicas são comuns a ambos. No entanto, a remoção do solvente durante a consolidação do produto na colagem de barbotina envolve a ação capilar de um molde poroso, enquanto que na colagem de folhas o solvente é evaporado. Também o tamanho e a forma dos produtos manufaturados pelos dois processos diferem consideravelmente.

Outros métodos como prensagem, compactação por rolos (roll compaction) e extrusão são usados para formar peças cerâmicas de espessura fina. Embora sejam mais "tradicionais", tais processos apresentam limitações quanto às dimensões das peças (folhas muito finas, menores que $0,25 \mathrm{~mm}$, não podem ser fabricadas facilmente), à qualidade da superfície do produto final, e ao investimento em equipamentos necessários para o processamento $[1,2]$.

As características da barbotina influenciam o arranjo das partículas no corpo a verde, o que, por sua vez, determina o comportamento da sinterização e as propriedades finais da peça fabricada. Assim, a barbotina usada na colagem de folhas cerâmicas tem que ser homogênea e estável, e essas características tem 


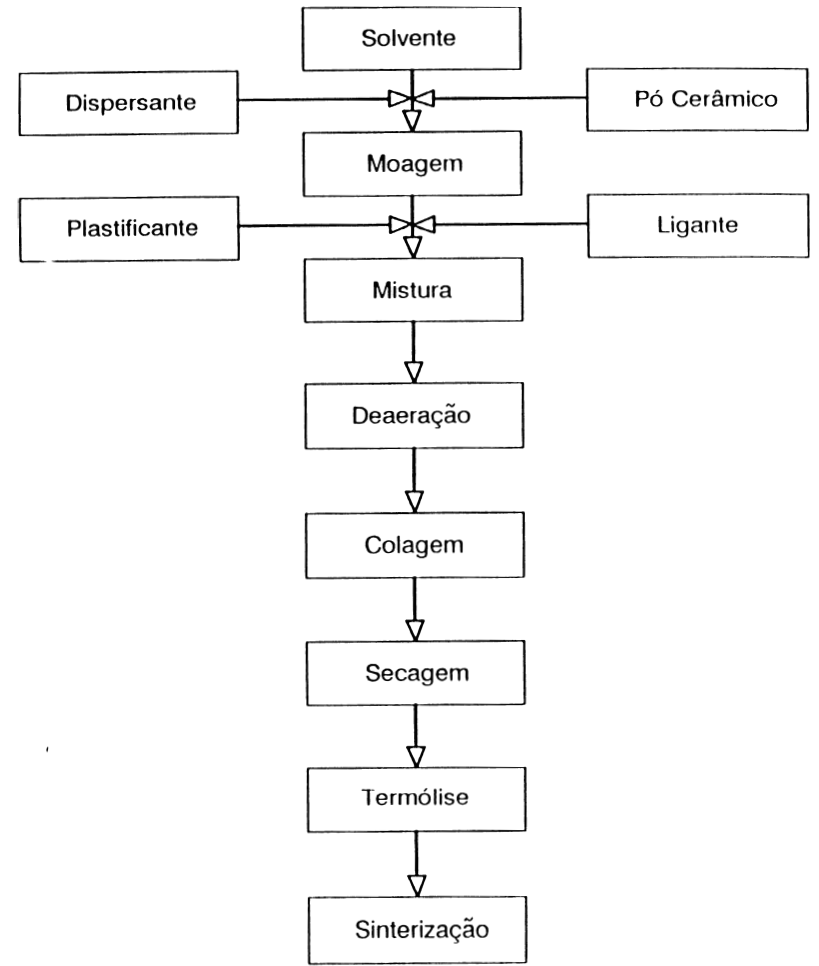

Figura 1: Fluxograma do processo de colagem de folhas cerâmicas.

que ser preservadas durante todas as etapas do processamento. Portanto, para se obter um produto de boa qualidade, é necessário inicialmente o entendimento dos mecanismos de estabilização e das características reológicas da barbotina.

\section{ESTABILIZAÇÃO DA BARBOTINA}

A estabilidade de uma suspensão com respeito à coagulação (floculação) é determinada pelas forças de atração e repulsão entre as partículas, o que depende de suas características físico-químicas e da interface partícula-solvente. A estabilidade da suspensão é atingida quando as forças repulsivas são suficientemente grandes para superar as forças de atração. Para se obter uma barbotina estável são usados dispersantes (defloculantes) que, adsorvidos pelas partículas, aumentam a magnitude da repulsão.

A força de atração está sempre presente, devido à tendência das partículas de estarem em contato umas com as outras através da força de van der Waals, que é função basicamente das propriedades dielétricas das partículas e do solvente. A interação repulsiva é fundamentalmente produzida por dois mecanismos diferentes. Um é a repulsão eletrostática, como resultado da formação de uma dupla camada elétrica em torno de cada partícula de pó disperso em um líquido polar. Um outro mecanismo é a estabilização estérica, na qual polímeros de cadeias longas adicionados à barbotina são adsorvidos sobre a superfície da partícula.

\section{Estabilização Eletrostática}

A estabilização eletrostática é conseqüência da presença de uma dupla camada de íons adsorvidos em partículas carregadas eletricamente, dispersas em um líquido polar (água ou solvente orgânico). Essa dupla camada consiste de uma camada fixa de íons de carga contrária à da superfície da partícula (camada de Stern) e de uma camada móvel de íons (camada difusa) [17], como mostrado na Fig. 2.

A diferença de potencial entre a camada difusa e a dispersão eletricamente neutra (potencial zeta, $\zeta$ ) pode ser determinada experimentalmente. $\mathrm{O}$ potencial zeta é um parâmetro importante para a

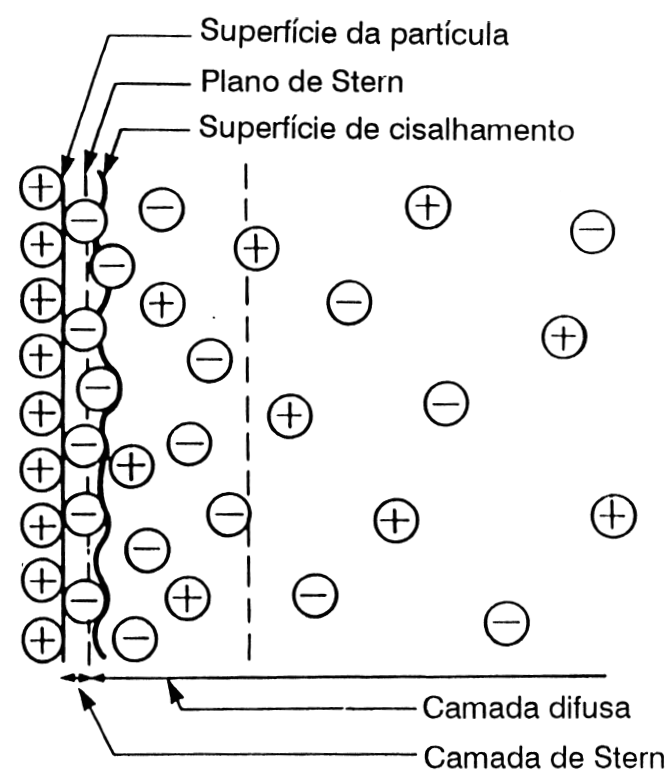

Figura 2: Esquema da dupla camada em uma superficie de carga positiva [17].

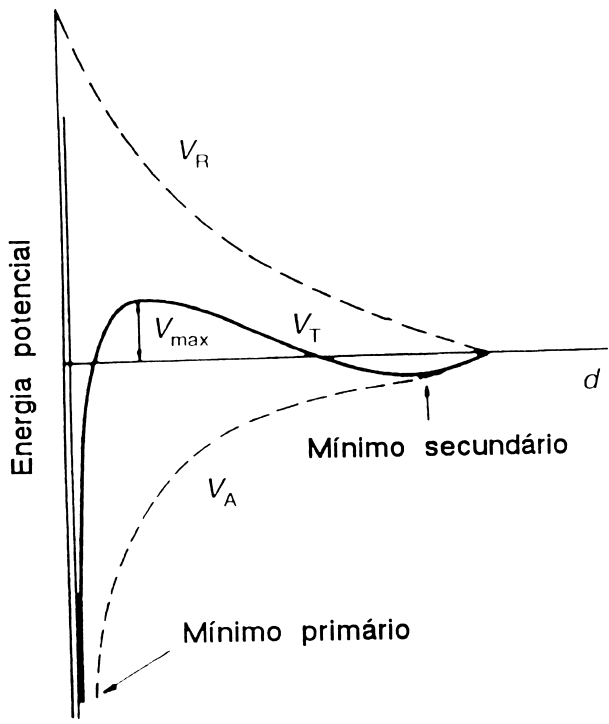

Figura 3: Energia potencial de interação entre duas partículas (repulsão eletrostática) [22].

avaliação da estabilidade de uma barbotina [18, 19]: se dois sistemas de diferentes valores de $\zeta$ forem comparados, permanecendo todos os outros fatores iguais, espera-se que o sistema que apresenta o maior valor de $\zeta$ seja mais estável em relação à floculação.

Uma suspensão é estável termodinamicamente quando o valor da energia potencial de interação entre as partículas, $V_{T}$, é negativo. $V_{T}$ é a resultante da soma da energia de atração, $V_{A}$, devida às forças de van der Waals, e da energia de repulsão, $V_{R}$, originada na 
dupla camada elétrica ao redor da partícula. Curvas de energia potencial como função da separação entre as partículas, $d$, têm sido quantificadas para a repulsão eletrostática pela teoria proposta por Derjaguin e Landau [20], Verwey e Overbeeck [21], a assim chamada teoria DLVO.

A Fig. 3 mostra a interação de duas partículas de acordo com a teoria DLVO para o mecanismo de estabilização eletrostática [22]. À medida que as partículas se aproximam, é atingido um ponto de metaestabilidade, correspondente a um mínimo secundário. Nesse estado, as partículas se mantêm separadas por um filme líquido a uma distância relativamente grande, ou seja, não há floculação. As partículas podem, no entanto, se vencida uma barreira de energia, $V_{\max }$, atingir um estado termodinamicamente mais estável, correspondente a um mínimo primário. Nesse ponto, a distância entre as partículas é a menor possível, o que significa na prática o fenômeno da floculação. A barreira de energia deve, portanto, ser suficientemente alta a fim de manter a suspensão num estado de relativa estabilidade eletrostática, no ponto de mínimo secundário. Fatores controláveis que influenciam esse processo de estabilização são o valor do potencial zeta, a concentração de íons na solução e o tamanho de partícula [23].

\section{Estabilização Estérica}

A estabilização estérica é conseqüência da interação física de substâncias poliméricas de cadeia longa, que são adsorvidas por partículas dispersas em uma barbotina. Com a aproximação das partículas, as camadas adsorvidas se interpenetram e as cadeias poliméricas tendem a se ordenar paralelamente (Fig. 4). Desta maneira, no equilíbrio termodinâmico, as partículas permanecem isoladas.

O sucesso da estabilização estérica depende das características superficiais da partícula, da configuração do polímero adsorvido e da espessura da camada adsorvida [3]. Tem sido provado experimentalmente que, para uma estabilização estérica efetiva, é necessário um polímero com uma massa molecular maior que $10000 \mathrm{~g} /$ mol [24]. Algumas moléculas, como ácido oléico ou esteárico, podem estabilizar uma suspensão também por adsorção, mas não têm uma cadeia carbônica longa o suficiente para prover uma estabilização estérica. Esse mecanismo é chamado de estabilização semiestérica [25] e tem sido efetivo em muitas formulações de barbotinas.

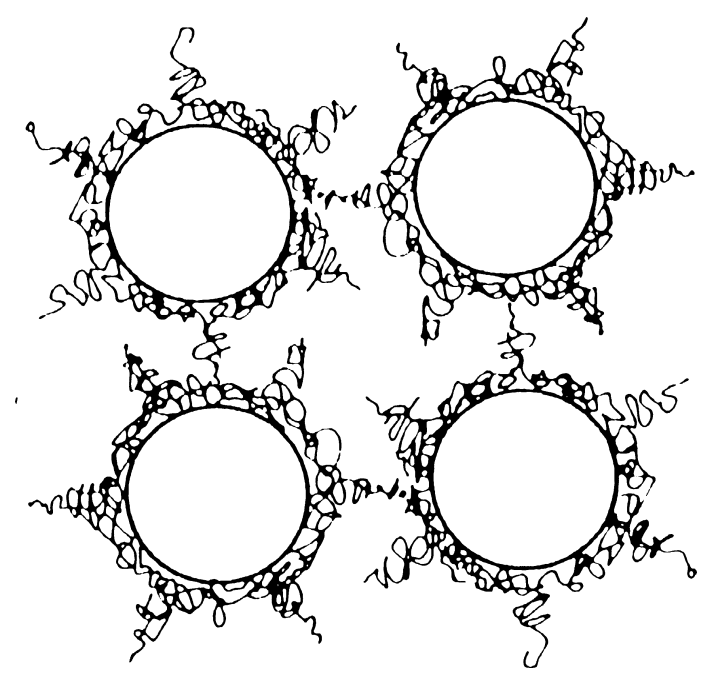

Figura 4: Mecanismo de estabilização estérica.

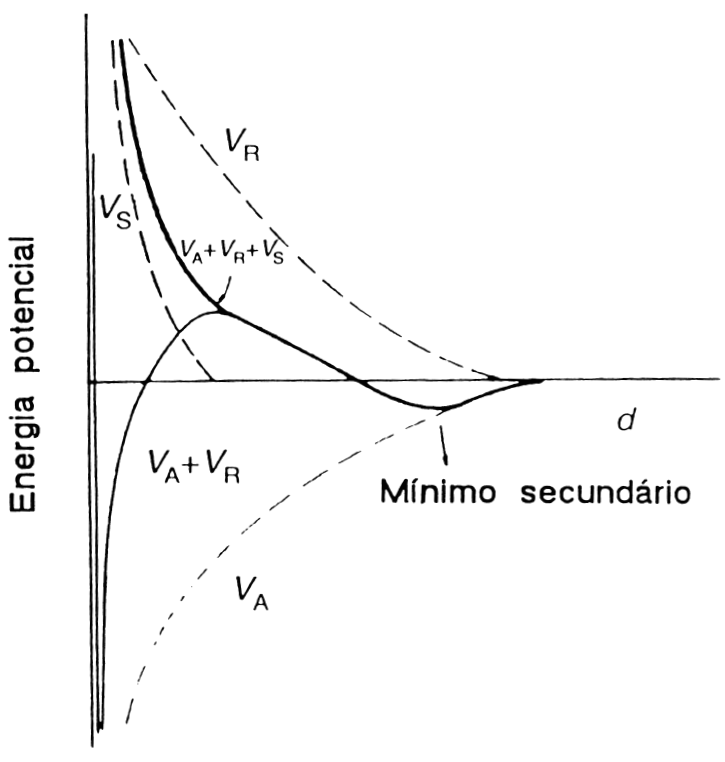

Figura 5: Energia potencial de interação entre duas partículas (repulsão eletroestérica) [22].

\section{Estabilização Eletroestérica}

A repulsão eletrostática é mais efetiva em água do que em líquidos não-aquosos, por causa da baixa constante dielétrica destes. A repulsão estérica, por sua vez, é efetiva tanto em meios aquosos quanto em não-aquosos. Pós estabilizados estericamente podem ser termodinamicamente estáveis, enquanto que pós estabilizados eletrostaticamente são apenas metaestáveis [22].

Uma combinação dos mecanismos eletrostático e estérico pode resultar uma melhor estabilização. Essa combinação se denomina estabilização eletroestérica [26]. O componente eletrostático pode ser originado de uma carga sobre a superfície da partícula dispersa e/ou por cargas associadas ao polímero adsorvido. Tais polímeros, que apresentam grupos ionizáveis repetidos, são chamados polieletrólitos. Polieletrólitos usados comumente são sais de ácidos tânicos e húmicos, e ácidos policarboxílicos [26, 27].

$\mathrm{Na}$ curva de energia potencial para este mecanismo combinado (Fig. 5), a dupla camada provê uma barreira de energia potencial alta a longas distâncias e a estabilização estérica, correspondente à curva $V_{S}$, previne o contato entre as partículas a curtas distâncias [22].

\section{REOLOGIA DA BARBOTINA}

Uma barbotina com boas características reológicas deve ter baixa viscosidade para facilitar o processo de colagem, mas não tão baixa a ponto de haver sedimentação. Por outro lado, para se obter maior densidade a verde após a colagem, a carga de sólidos deve ser relativamente alta, de modo a se reduzir a retração final.

Na colagem de folhas cerâmicas, a medida da viscosidade da barbotina é um fator importante para o controle do processo. O comportamento reológico de suspensões pode ser descrito por vários modelos. Um modelo que se aplica em geral é o de Ostwald, no qual a dependência da tensão de cisalhamento $\tau$ em relação à taxa de cisalhamento $\gamma$ é dada através de uma constante $A$ e do 
expoente de Ostwald $n$ [28]:

$$
\tau=A \gamma^{n}
$$

O comportamento de escoamento é, para $n>1$, do tipo dilatante, para $n=1$, newtoniano e, para $n<1$, pseudoplástico.

A viscosidade dinâmica, $\eta$, é, por sua vez, uma constante somente para líquidos newtonianos, definida por:

$$
\eta=\tau / \gamma
$$

Para fluidos não-newtonianos, como é em geral a barbotina usada em colagem de folhas, pode-se falar de uma viscosidade aparente para um determinado ponto, que é função de $\tau$ e $\gamma$. Além disso, sistemas floculados apresentam freqüentemente um limite de escoamento $\tau_{0}$, ou seja, só começam a escoar quando submetidos a tensões de cisalhamento $\tau>\tau_{0}$. Para tais sistemas, pode-se aplicar o modelo de escoamento de Herschel-Bulkley [28], análogo ao modelo de Ostwald:

$$
\tau=\tau_{0}+A \gamma^{n} \quad(\mathrm{C})
$$

A Fig. 6 mostra curvas de forma esquemática para diferentes modelos de escoamento.

A reologia da barbotina é determinada pelas características dos seus componentes. Os ligantes, por exemplo, aumentam a vis-

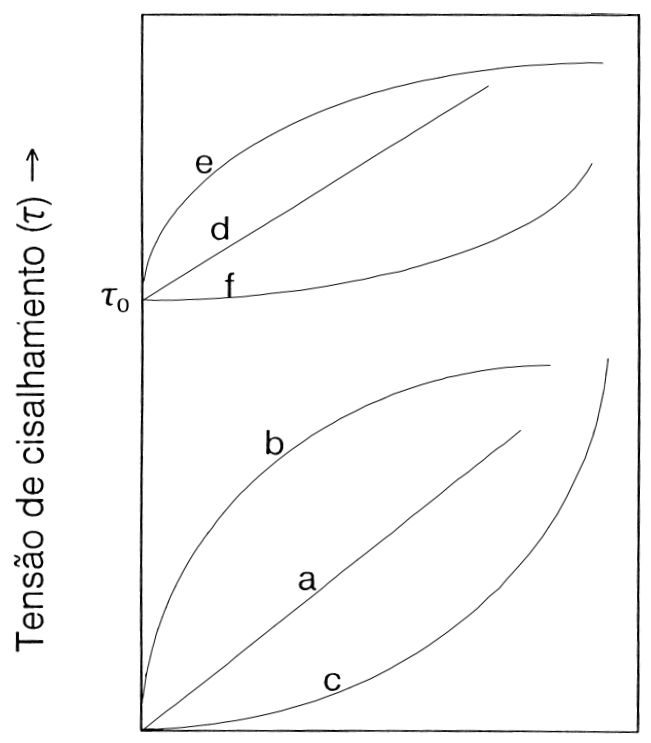

Taxa de cisalhamento $(\Upsilon) \rightarrow$
(a) newtoniano
(b) pseudoplástico
(c) dilatante
(d) newtoniano com limite de escoamento $\tau_{0}$
(e) pseudoplástico com $\tau_{0}$
(f) dilatante $\operatorname{com} \tau_{0}$

Figura 6: Modelos de escoamento.

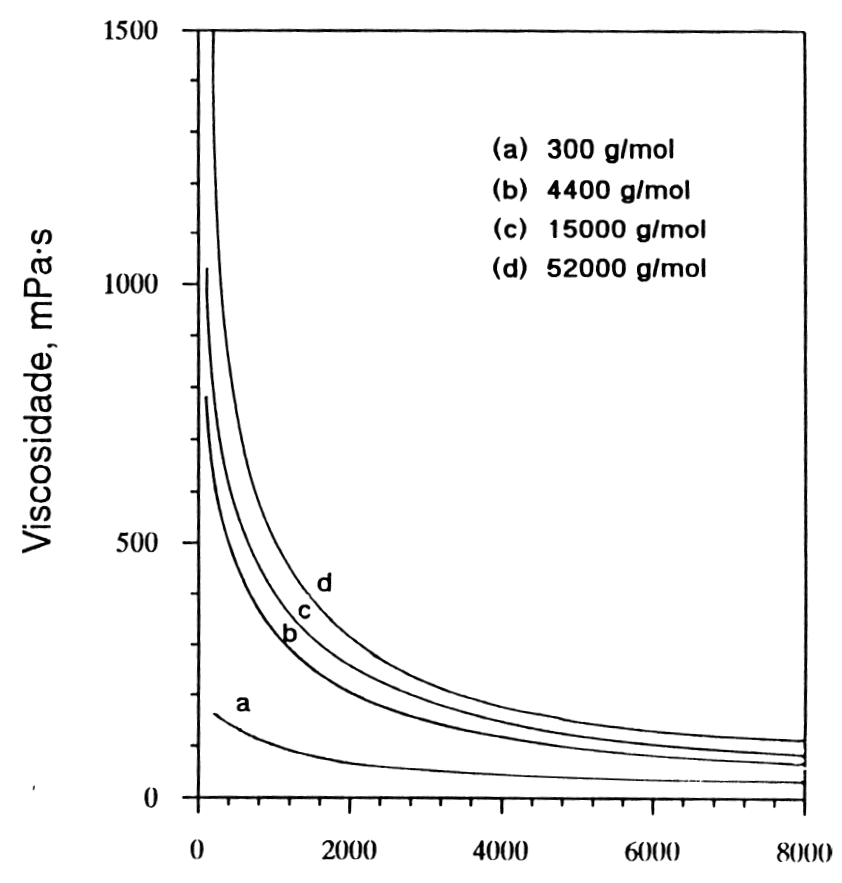

Taxa de cisalhamento, $\mathrm{s}^{-1}$

Figura 7: Viscosidade em função da taxa de cisalhamento para HEC 2\%, com diferentes massas moleculares, a $25^{\circ} \mathrm{C}$ [30].

cosidade e mudam o comportamento de escoamento de newtoniano para pseudoplástico. Valores típicos de viscosidade para barbotinas no processo de colagem de folhas cerâmicas estão na faixa de 5 a $25 \mathrm{~Pa}$.s para gradientes de velocidade de até $1000 \mathrm{~s}^{-1}$ [29]. A Fig. 7 mostra uma relação viscosidade-taxa de cisalhamento para uma solução $2 \%$ em massa de hidroxietilcelulose, um ligante fortemente pseudoplástico [30]. Como se pode observar, a viscosidade é alta próximo ao estado de repouso e decresce com a velocidade de escoamento. Dessa maneira, durante o processo de colagem, uma barbotina pseudoplástica escoa com facilidade no início e, assim que o filme é produzido, a viscosidade aumenta e a superfície do filme se mantém nivelada, antes da evaporação do solvente. Modelos analíticos do comportamento do fluxo de uma barbotina cerâmica têm sido apresentados na literatura [31,32].

\section{FORMULAÇÃO DA BARBOTINA}

As características da barbotina são naturalmente determinadas pelas propriedades de seus constituintes (matriz cerâmica, solvente, dispersante, ligante e plastificante) e da interação entre eles. Inúmeras composições de barbotina usada em colagem de folhas têm sido relatadas na literatura [29]. A seguir, são apresentados alguns conceitos básicos relativos aos componentes da barbotina.

\section{Pós Cerâmicos}

Vários pós têm sido utilizados na fabricação de peças cerâmicas via colagem de folhas, como $\mathrm{BaTiO}_{3}$ [3-5], $\mathrm{Al}_{2} \mathrm{O}_{3}$ [6-11], AlN [10-13], entre outros. Em alguns casos, são acrescentados aditivos de sinterização, como $\mathrm{CaO}, \mathrm{MgO}$ ou $\mathrm{Y}_{2} \mathrm{O}_{3}$ para $\mathrm{Al}_{2} \mathrm{O}_{3}$ ou AlN; e inibidores de crescimento de grão, tais como $\mathrm{ZrO}_{2}$ ou SiC para $\mathrm{Al}_{2} \mathrm{O}_{3}$ [33]. A quantidade de pó cerâmico na barbotina varia 
em geral de 40 a $50 \%$ em massa [34].

Os parâmetros importantes que devem ser controlados num pó cerâmico usado na colagem de folhas são o tamanho médio de partícula e sua distribuição, a área superficial e o nível de impurezas. Em geral, tamanhos de partícula entre 1 e $4 \mu \mathrm{m}$ e áreas de superfície específica entre 2 e $6 \mathrm{~m}^{2} / \mathrm{g}$ têm sido usados para substratos de alumina $[8,35]$.

\section{Solventes}

Os solventes são usados na colagem de folhas para dissolver os componentes orgânicos adicionados à barbotina (dispersantes, ligantes e plastificantes). Eles devem ser quimicamente inertes em relação aos pós cerâmicos utilizados, ter um baixo ponto de ebulição (termólise a temperaturas mais baixas) e uma baixa viscosidade (melhor escoamento na colagem). Como solventes podem ser usados água ou líquidos orgânicos, como etanol, isopropanol, acetona, e metiletilcetona, em concentrações normalmente de 30 a 50\% em massa [34].

A maior parte das práticas comerciais de fabricação usa sistemas não-aquosos, que oferecem as vantagens de ter um baixo ponto de ebulição e de evitar a hidratação dos pós cerâmicos. Por outro lado, solventes orgânicos requerem precauções especiais com respeito a toxidez e inflamabilidade [36]. Devido a considerações ambientais e de saúde, o uso de solventes orgânicos tem sido questionado nos últimos anos e muitas formulações de barbotina em base aquosa têm sido empregadas [37].

Uma comparação entre barbotina aquosa e não-aquosa, mantidas as demais variáveis do sistema tão similares quanto possível, foi realizada [38]. Os resultados indicaram que as folhas a verde têm propriedades semelhantes, embora o sistema não-aquoso tenha sido relativamente insensível a perturbações no processo. Problemas associados ao uso de sistemas aquosos têm sido verificados [36], tais como coagulação do pó na barbotina, formação de espuma durante a moagem, polimerização prematura, sedimentação da barbotina, dificuldade de se obter altas densidades a verde, entre outros. Uma desvantagem adicional dos sistemas aquosos seria a impossibilidade de se usar pós cerâmicos que reagem com água. No entanto, o tratamento superficial de pós reativos, como AlN, permite o seu processamento em meio aquoso $[39,40]$.

\section{Dispersantes}

Os dispersantes (defloculantes) cobrem as partículas cerâmicas e as mantêm numa suspensão estável na barbotina. A interação repulsiva entre as partículas é provida por meio de mecanismos eletrostáticos e/ou estéricos, dependendo das características físicoquímicas dos dispersantes adicionados. Deve-se usar tanto quanto possível uma pequena concentração de dispersante (por exemplo, cerca de $3 \mathrm{~mL}$ de dispersante por $100 \mathrm{~g}$ de pó cerâmico) [2], já que este tem que ser eliminado na termólise, sem deixar resíduos no corpo sinterizado.

Há um valor ótimo de dispersante que proporciona viscosidade mínima e densidade a verde máxima, que pode ser determinado experimentalmente [4]. Esse valor pode ser obtido não só por medidas de viscosidade, como também por medidas de adsorção ou potencial zeta, como por exemplo para concentrações variáveis de éster do ácido fosfórico em etanol (Fig. 8) [41].

Como dispersantes em barbotinas não-aquosas são empregados comumente éster do ácido fosfórico e óleo de peixe, os quais,

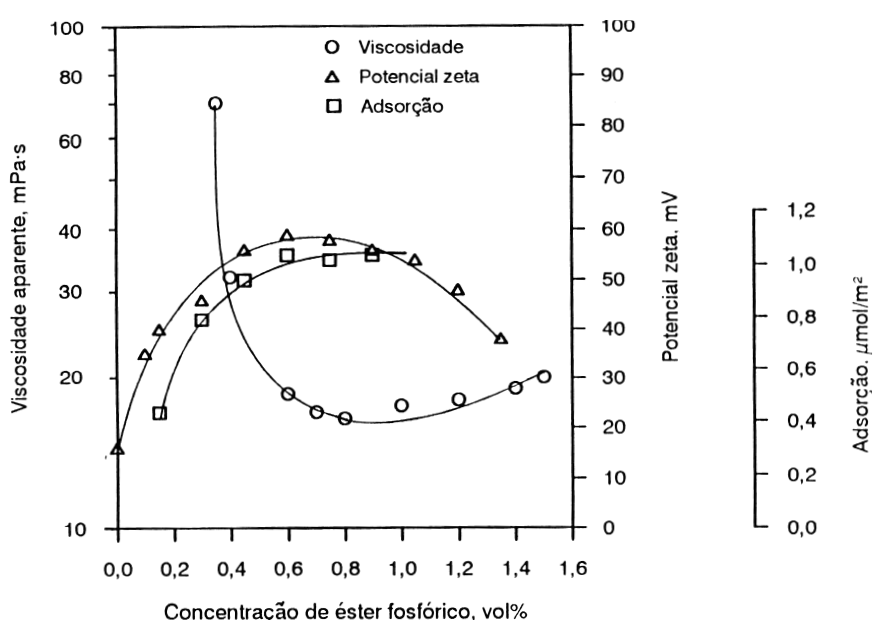

Figura 8: Viscosidade, adsorção e potencial zeta como função da concentração de éster do ácido fosfórico [41].

em comparação com outras substâncias, têm um efeito de dispersão adequado já em pequenas quantidades [41]. No caso de barbotinas de base aquosa, têm sido utilizados como meio de dispersão principalmente polieletrólitos do tipo sal de amônio e o sal de sódio de poliácidos carboxílicos [36].

\section{Ligantes}

Os ligantes são adicionados a barbotinas para se obter uma folha cerâmica com resistência mecânica a verde suficiente para permitir sua manipulação e armazenagem. $\mathrm{O}$ ligante forma pontes orgânicas entre as partículas que resultam numa adesão forte depois da evaporação do solvente. Os ligantes devem ser compatíveis com o sistema, auxiliar a estabilização, atuar como lubrificante entre as partículas, se decompor a baixas temperaturas sem deixar resíduos, e ser efetivos a baixas concentrações (abaixo de $10 \%$ em massa) [8].

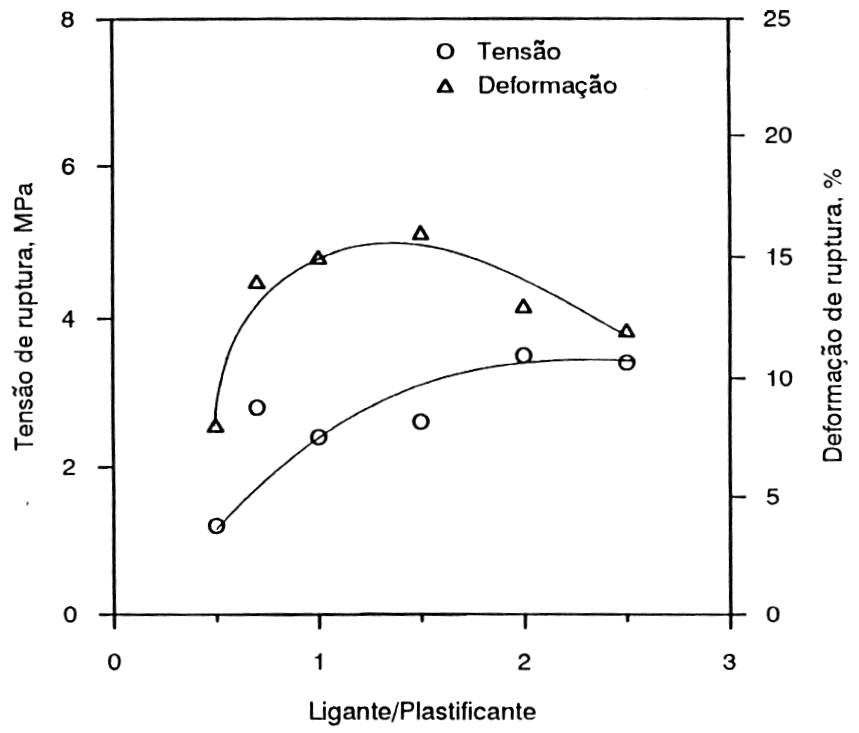

Figura 9: Tensão e deformação de ruptura em relação à razão ligante plastificante [42]. 
Há muitos tipos de substâncias, naturais ou sintéticas, usadas como ligantes na colagem de folhas. Em geral, em barbotinas aquosas são usados ligantes derivados da celulose, como metilcelulose (MC), hidroxietilcelulose (HEC) e carboximetilcelulose (CMC), ou ainda ligantes do tipo vinil, como polivinilálcool (PVA) ou polivinilacetato (PVAc). Outros ligantes do tipo vinil são usados em líquidos não-polares, como polivinilbutiral (PVB) ou polimetilmetacrilato (PMMA) [29].

\section{Plastificantes}

Os plastificantes dissolvem os ligantes e melhoram a distribuição destes na barbotina, o que causa a flexibilidade da folha cerâmica a verde. Através da seleção adequada do sistema ligante/ plastificante e do ajuste das concentrações relativas é possível variar a flexibilidade da folha. Os plastificantes são, por outro lado, imprescindíveis para se melhorar a trabalhabilidade das folhas a verde, facilitando a separação dessas da superfície em que foram coladas. Os plastificantes mais usados são glicóis, como polietilenoglicol (PEG), glicerina, e ftalatos, como dibutilftalato (DBP).

A razão ótima ligante/plastificante pode ser determinada experimentalmente para cada sistema. Normalmente, o conteúdo de plastificante é menor ou igual ao conteúdo de ligante [2]. Por exemplo, no caso de uma barbotina não-aquosa de alumina [42], a folha a verde exibiu flexibilidade ótima para uma razão ligante/ plastificante de 1 a 1,5 (Fig. 9). Outros resultados experimentais mostram que a adição de plastificante aumenta a deformação sob tensão em detrimento da resistência à ruptura sob tensão [35].

\section{PROCESSO E EQUIPAMENTOS}

\section{Moagem, Mistura e Deaeração}

Primeiramente, o pó cerâmico é misturado a uma solução solvente mais dispersante e moído, de modo a se destruir aglomerados e se distribuir uniformemente o agente dispersante sobre a superfície das partículas, formando uma barbotina de baixa viscosidade. Posteriormente, ocorre uma etapa de mistura e homogeneização, na qual o plastificante e o ligante são dissolvidos na barbotina, aumentando a viscosidade desta.

A moagem/mistura, tipicamente de 24 a 48 horas no total [1], é feita em equipamento padrão de cominuição, como moinho de bola ou vibratório. Deve-se usar esferas ou rolos que não reajam com o solvente empregado nem contaminem os materiais sendo moídos e misturados.

A ordem em que os componentes da barbotina são adicionados é muito importante. Deve-se introduzir o dispersante ao solvente antes dos outros aditivos, de modo a prevenir a competição pela superfície das partículas cerâmicas e, assim, produzir uma barbotina mais uniforme $[8,43]$. A adição de plastificantes antes da adição de ligantes auxilia a dissolução do ligante na barbotina [36]. Acredita-se que isso se deve à solubilidade preferencial do ligante no plastificante em relação à sua solubilidade no solvente.

$\mathrm{Na}$ moagem/mistura ocorre freqüentemente a formação de espuma na barbotina, que precisa ser eliminada antes da colagem. Para tanto, faz-se uso de um equipamento específico, como uma câmara de deaeração a vácuo, ou de uma etapa de rolagem lenta ou ainda centrifugação. Depois da barbotina ter sido deaerada, ela está pronta para ser transferida para o equipamento de colagem. Em

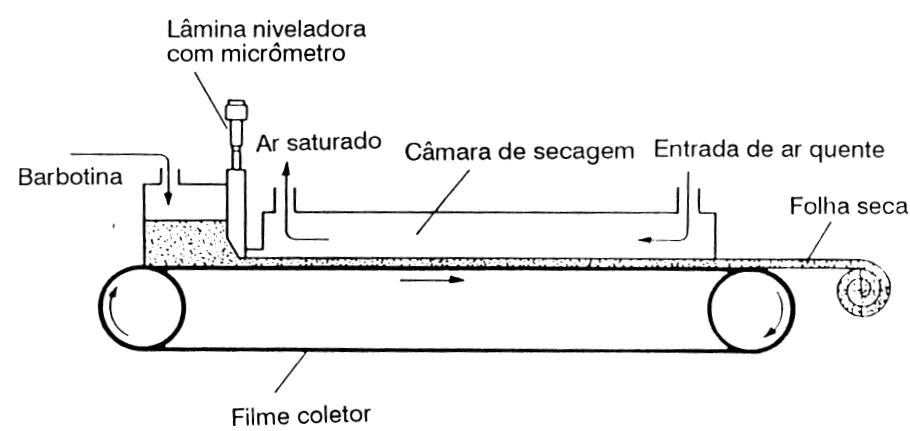

Figura 10: Equipamento de processamento contínuo de colagem de folhas cerâmicas [35].

alguns processos, ela é bombeada, em outros, é conduzida por gravidade. Um filtro é comumente utilizado para remover aglomerados remanescentes [44]. A abertura do filtro usado depende do tamanho de partícula de pó disperso e da viscosidade da barbotina.

\section{Colagem}

A colagem de folhas propriamente dita é efetuada através do movimento relativo entre uma lâmina niveladora (doctor blade) $\mathrm{e}$ uma superfície coletora. Duas soluções são possíveis: ou a lâmina se move sobre uma superfície fixa (processo descontínuo), ou a superfície se move sob uma lâmina fixa (processo contínuo). A maior parte da produção em grande escala é baseada na colagem contínua. Para a manufatura em pequena escala, ou para experimentos em laboratório, o processo descontínuo pode ser usado.

Equipamentos de colagem têm sido construídos com câmaras de colagem de 10 a $125 \mathrm{~cm}$ de largura, e de 2 a $38 \mathrm{~m}$ de comprimento [36]. O equipamento padrão industrial (Fig. 10) consiste de uma superfície móvel de colagem, um sistema de secagem por fluxo de ar quente, um dispositivo com as lâminas, e um sistema de separação da folha [35]. Para se prover uma superfície coletora limpa, livre de defeitos são geralmente usados, no processo descontínuo, placas de vidro, ou, no processo contínuo, uma lâmina de aço inox contínua, sobre a qual podem ser adaptados filmes poliméricos de teflon, polietilieno ou acetato de celulose [1].

Para se regular precisamente a espessura da folha a ser produzida, a altura da lâmina em relação à superfície é ajustada por meio de micrômetros. Um sistema dual de lâminas tem sido empregado para um controle mais preciso da operação de colagem (Fig. 11) [8]. Outros parâmetros, como a viscosidade da barbotina, a velocidade do dispositivo móvel na colagem, e a pressão exercida pela barbotina no reservatório também influenciam a espessura do

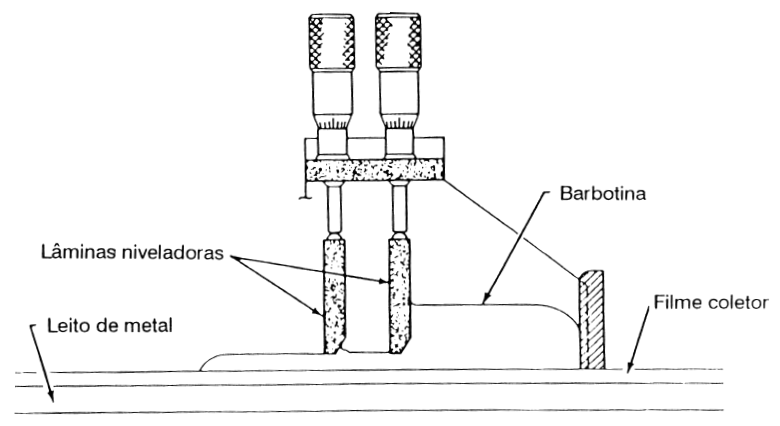

Figura 11: Dispositivo de lâminas duplas para colagem de folhas cerâmicas [8]. 
filme colado [31]. A velocidade de colagem em equipamentos contínuos varia de 5 a $100 \mathrm{~cm} / \mathrm{min}[2,36]$, dependendo das condições de secagem e da velocidade de produção requerida.

\section{Etapas Pós-colagem}

A secagem da folha colada é uma etapa importante no processamento. Uma secagem rápida demais da barbotina úmida faz com que a superfície se feche, impedindo a remoção do solvente e causando a formação de poros na folha. Tal problema pode ser evitado através do princípio da secagem a úmido: introduz-se na câmara de colagem e/ou secagem uma determinada quantidade do solvente utilizado [2]. Dessa forma, de acordo com o equilíbrio líquido-vapor, se permite a migração lenta do solvente para a superfície da folha colada.

O encolhimento na direção da espessura da folha após a secagem é usualmente cerca de 50\% em relação à espessura nivelada pelas lâminas na colagem [7]. Uma modelagem do estágio de secagem pode ser encontrado na literatura [8]. Um fluxo de ar quente contracorrente à direção de colagem é geralmente usado para se acelerar o processo de secagem. Depois de seca, a folha pode ser ou enrolada num cilindro ou cortada em pequenas placas para uso em etapas posteriores. Nesse ponto, a folha é usualmente sujeita ao controle de qualidade, para características como densidade a verde ou acabamento superficial.

Em pacotes cerâmicos de multicamadas, a próxima etapa envolve a usinagem de furos nas folhas. Em capacitores de multicamadas, as folhas são metalizadas por meio de eletrodos. Após a metalização, as folhas cerâmicas são prensadas numa estrutura laminada. Temperaturas desde a ambiente até $110{ }^{\circ} \mathrm{C}$ e pressões na faixa de 1,38 a $138 \mathrm{MPa}$ são comumente usadas na laminação [36].

A eliminação dos componentes orgânicos (ligantes, plastificantes, dispersantes) por tratamento térmico é freqüentemente feita por aquecimento lento ao ar [45], em temperaturas relativamente baixas $\left(<500^{\circ} \mathrm{C}\right)$ [36]. A termólise pode ocorrer através de diferentes mecanismos, como difusão, no caso, por exemplo, de resinas acrílicas, ou por meio de forças capilares, quando os componentes orgânicos se fundem, como PVB-DBP [46]. Em qualquer caso, a velocidade de aquecimento precisa ser controlada, de modo que a taxa de evaporação dos componentes orgânicos não ultrapasse a taxa de difusão (ou de transporte capilar). Do contrário, são formadas bolhas de vapor dentro do corpo a verde que, por sua vez, levam à formação de trincas [47].

Depois da eliminação dos componentes orgânicos, as folhas cerâmicas são sinterizadas. No caso das cerâmicas de multicamadas, é feita a chamada cossinterização, na qual a folha cerâmica e a parte metalizada são simultaneamente sinterizadas. Por exemplo, para pacotes de multicamadas à base de alumina metalizada com molibdênio ou tungstênio, a sinterização ocorre acima de $1600{ }^{\circ} \mathrm{C}$ em atmosfera redutora (hidrogênio ou nitrogênio/hidrogênio) [48]. Depois da sinterização , o pacote cerâmico ou o capacitor de multicamadas está pronto para uma etapa final de metalização e/ou para a adaptação de pinos ou de chips semicondutores.

\section{CONSIDERAÇÕES FINAIS}

A seleção de aditivos apropriados para a barbotina é a chave para se obter um produto final de boa qualidade. Para tanto, devese conhecer os mecanismos de atuação desses aditivos e de interação entre eles e as partículas cerâmicas na suspensão. Especialmente importantes são os conceitos de estabilização de uma barbotina e as suas propriedades reológicas.

Atualmente, a colagem de folhas cerâmicas é o processo básico de fabricação de estruturas de multicamadas para a indústria eletrônica. Proporcionalmente à crescente tendência à miniaturização e ao uso de pacotes de multicamadas, deve ocorrer a difusão e o aperfeiçoamento do processo de colagem de folhas cerâmicas.

\section{AGRADECIMENTOS}

$\mathrm{O}$ autor agradece ao $\mathrm{CNPq} / \mathrm{RHAE}$ pelo suporte financeiro.

\section{REFERÊNCIAS}

[1] R. E. Mistler, Tape Casting in Ceramic and Glasses. Engineered Materials Handbook. v. 4, Ed.: J. S. Schneider Jr, Materials Park, ASM International, 1991. p. 161-5.

[2] E. P. Hyatt, Making Thin, Flat Ceramics -3/4 A Review. Am. Ceram. Soc. Bull. 65 (4): 637-8, 1986.

[3] K. Mikeska, W. R. Cannon, Dispersants for Tape Casting Pure Barium Titanate. In: MANGELS, J. A.; MESSING, G. L. (Ed.) Forming of Ceramics. Advances in Ceramics. v. 9. Columbus $(\mathrm{OH})$, Am. Ceram. Soc., 1984. p. 164-83.

[4] A. Karas, T. Kumagai, W. R. Cannon, Casting Behaviour and Tensile Strength of Cast Barium Titanate Tapes as the Organic Composition is Varied. Adv. Ceram. Mater. 3 (4): 374-7, 1988.

[5] I. Burn, Ceramic Capacitor Dielectrics. In: SCHNEIDER, S. J., Jr. (Ed.) Ceramic and Glasses. Engineered Materials Handbook. v. 4. Materials Park, ASM International, 1991. p. 1112-8.

[6] D. J. Shanefield, R. E. Mistler, Manufacturing Process for FineGrained Alumina Substrates: the Am. Ceram. Soc. Bull. 52 (4): $375,1973$.

[7] J. C. Williams, Doctor-Blade Process. In: Wang, F. F. Y. (Ed.) Ceramic Fabrication Processes. Treatise on Materials Science and Technology. v. 9. New York, Academic Press, 1976. p. 173-98.

[8] R. E. Mistler, D. J. Shanefield, R. B. Runk, Tape Casting of Ceramics. In: G. Y. Onoda, L. L. Hench, (Ed.) Ceramic Processing Before Firing. New York, John Wiley, 1978. p. 411-48.

[9] D. Cooper, P. G. Newland, F. W. Shapley, The Development of High-Quality Alumina Substrates. In: P. Vicenzini, (Ed.) High Tech Ceramics. Amsterdam, Elsevier, 1987. p. 1549-54.

[10] D. G. Wirth, Ceramic Substrates. In: S. J. Schneider Jr., (Ed.) Ceramic and Glasses. Engineered Materials Handbook. v. 4. Materials Park, ASM International, 1991. p. 1107-11.

[11] R. R. Tummala, M. R. Haley, G. Czornyj, Materials in Microelectronics. Ceramics International 19: 191-210, 1993.

[12] D. G. Brunner, Foliengießen, Sintern und Anwendungen von Aluminiumnitrid. Sprechsaal 121 (3): 181-6, 1988.

[13] E. Streicher, T. Chartier; P. Boch, Influence of Organic Components on Properties of Tape-Cast Aluminium Nitride Substrates. Ceram. Int. 16 (4) 247-52, 1990.

[14] G. N. Howatt, R. G. Breckenridge, J. M. Brownlow, Fabrication of Thin Ceramic Sheets for Capacitors. J. Am. Ceram. Soc. 30: 237-42, 1947.

[15] G. N. Howatt, Method of Producing High Dielectric, High Insulation Ceramic Plates. U. S. Patent 2.582.993, 1952.

[16] J. L. Parks Jr., Manufacture of Ceramics. U. S. Patent 
2.966.719, 1961 .

[17] D. J. Shaw, Introduction to Colloid and Surface Chemistry. 4 ed. London, Butherworth-Heinemann, 1992. p. 174-209.

[18] R. J. Hunter, Zeta Potential in Colloid Chemistry. New York, Academic Press, 1981.

[19] W. R. Cannon, R. Becker, K. R. Mikeska, Interactions Among Organic Additives for Tapecasting In: M. F. Yan, K. Niwa, H. M. O’Bryan Jr., W. S. Young, (Ed.) Ceramic Substrates and Packages for Electronic Applications. Advances in Ceramics. v. 26. Westerville (OH), Am. Ceram. Soc. 1986. p. 525-41.

[20] B. V. Derjaguin, J. D. Landau, Acta Physicochim. USSR 14: $633,1941$.

[21] E. J. W. Verwey, J. T. G. Overbeeck, Theory of the Stability of Lyophobic Colloids. Amsterdam, Elsevier, 1948.

[22] R. Moreno, The Role of Slip Additives in Tape Casting Technology: Part I -3/4 Solvents and Dispersants. Am. Ceram. Soc. Bull. 71 (10): 1521-31, 1992.

[23] R. Hogg, Flocculation Phenomena in Fine Particle Dispersions. In: G. L. Messing, (Ed.) Ceramic Powder Science. Advances in Ceramics. v. 21. Westerville (OH), Am. C eram. Soc. 1987. p. 467-81. [24] D. H. Napper, Polymeric Stabilization of Colloidal Dispersions. New York, Academic Press, 1983.

[25] R. E. Johnson Jr., W. H. Morrison Jr., Ceramic Powder Dispersion in Nonaqueous Systems In: G. L. Messing, K. S. Mazdiyasni, J. W. McCauley, R. A. Haber, (Ed.) Ceramic Powder Science. Advances in Ceramics. v. 21. Westerville $(\mathrm{OH}), \mathrm{Am}$. Ceram. Soc., 1988. p. 325-48.

[26] J. Cesarano III, A. Aksay, A. Bleier, Stability of Aqueous $\alpha$ Alumina Suspensions with Poly (Methacrylic Acid) Polyelectrolyte. J. Am. Ceram. Soc. 71 (4): 250-5, 1988.

[27] R. Bast, On the Use of Dispersants and Deflocculants $c f i / B e r$. $D K G 67$ (9) 395-8, 1990.

[28] W. M. Kulicke, (Ed.) Fließverhalten von Stoffen und Stoffgemischen. Basel, Hüthig und Wepft, 1986.

[29] R. Moreno, The Role of Slip Additives in Tape Casting Technology: Part II -3/4 Binders and Plasticizers. Am. Ceram. Soc. Bull. 71 (11): 1647-57, 1992.

[30] G. Y. Onoda, The Rheology of Organic Binder Solutions. In: G. Y. Onoda, L. L. Hench, (ED.) Ceramic Processing Before Firing. New York, John Wiley, 1978. p. 235-51.

[31] Y. T. Chou, Y. T. Ko, Y. F. Man, Fluid Flow Model for Ceramic Tape Casting. J. Am. Ceram. Soc. 70 (10): C/280-2, 1987.

[32] T. A. Ring, A Model of Tapecasting Bingham and Newtonian Fluids. In: M. F. Yan, K. Niwa, Jr., H. M. O’Bryan; W. S. Young, (Ed.) Ceramic Substrates and Packages for Electronic Applications. Advances in Ceramics. v. 26. Westerville (OH), Am. Ceram. Soc. 1986. p. 569-82.

[33] A. Ezis, J. A. Rubin, Hot Pressing. In: S. J., Jr. Schneider, (Ed.) Ceramic and Glasses. Engineered Materials Handbook. v. 4. Materials Park, ASM International, 1991. p. 186-93.
[34] J. Heinrich, Folienguß. In: J. Kriegesmann, (Ed.) Technische keramische Werkstoffe. Köln, Deutscher Wirtschaftsdienst, 1991. p. 3.4.6.0/1-13.

[35] A. Roosen, Basic Requirements for Tape Casting of Ceramic Powders. In: G. L. Messing, E. R. Fuller, H. Hausner, (Ed.) Ceramic Powder Science II. Ceramic Transactions. v. 1. Westerville (OH), Am. Ceram. Soc., 1988, p. 675-92.

[36] R. E. Mistler, Tape Casting: The Basic Process for Meeting the Needs of the Electronics Industry. Am. Ceram. Soc. Bull. 69 (6): 1022-6, 1990.

[37] D. Hotza, P. Greil, Review: Aqueous Tape Casting of Ceramic Powders. Materials Science and Engineering A A202 (11): 20617, 1995.

[38] P. Nahass, W. E. Rhine, R. L. Pober, H. K. Bowen; W. L. Robbins, A COMPARISON OF AQUEOUS AND NONAQUEOUS SLURRIES FOR TAPE-Casting, and Dimensional Stability in Green Tapes. In: K. M. Nair, P. Pohanka, R. C. Buchanan, (Ed.) Materials and Processes for Microelectronic Systems. Ceramic Transactions. v. 15. Westerville (OH), Am. Ceram. Soc., 1990. p. 355-64.

[39] D. Hotza, O. Sahling; P. Greil, Hydrophobing of Aluminium Nitride Powders. Journal of Materials Science 30 (1): 127-32, 1995. [40] D. Hotza, N. Janssen, N. Claussen; P. Greil, Hydrophobic Aluminum Nitride Powder for Aqueous Tape Casting. Ceramic Transactions 51, 397-401, 1995.

[41] W. R. Cannon, J. R. Morris, K. R. Mikeska, Dispersants for Non-Aqueous Tape Casting. In: J. B. Blum, W. R. Cannon, (Ed.) Multilayer Ceramic Devices. Advances in Ceramics. v. 19. Westerville (OH), Am. Ceram. Soc. 1986. p. 161-74.

[42] B. Ryu, M. Takahasi, S. Suzuki, Effect of Added Organics on Mechanical Properties of Alumina Green Sheets Prepared by Doctor Blade Method. J. Ceram. Soc. Japan 100 (4): 422-5, 1992.

[43] L. Braun, J. L. Morris, W. R. Cannon, Viscosity of Tape Casting Slips. Am. Ceram. Soc. Bull. 64 (5): 727-9, 1985.

[44] D. J. Shanefield, R. E. Mistler, Filter for Ceramic Slips. Am. Ceram. Soc. Bull. 55 (2): 213, 1976.

[45] P. Boch, T. Chartier, Understanding and Improvement of Ceramic Processes: The Example of Tape Casting. Materials Science Forum 34-36: 813-9, 1988.

[46] J. A. Lewis, M. J. Cima, Direct Observations of Binder Distribution Processes in 2-D Porous Networks During Thermolysis. In: G. L. Messing; S. Hirano;H. Hausner, (Ed.) Ceramic Powder Science III. Ceramic Transactions. v. 12. Westerville (OH), Am. Ceram. Soc., 1990, p. 583-90.

[47] C. Dong, H. K. Bowen, Hot-Stage Study of Bubble Formation During Binder Burnout. J. Am. Ceram. Soc. 72 (7): 1192-9, 1989. [48] R. Röll, J. Brandenburger, Chip-Träger. In: KRIEGESMANN, J. (Ed.) Technische keramische Werkstoffe. Köln, Deutscher Wirtschaftsdienst, 1991, p. 8.5.1.0/1-14.

(Rec. 24/07/97, ac. 22/09/97) 